Qualitative-other

Hospital discharge recommendations can be confusing for people of low socioeconomic status, and difficult to follow due to constraints or conflicting personal goals

10.1136/eb-2014-101824

Jack Tsai

Department of Psychiatry, Yale University School of Medicine, West Haven, Connecticut, USA

Correspondence to: Dr Jack Tsai, Department of Psychiatry, Yale University School of Medicine, 950 Campbell Ave., 151D, West Haven, CT 06516, USA; Jack.Tsai@yale.edu

Commentary on: Kangovi S, Barg FK, Carter T, et al. Challenges faced by patients with low socioeconomic status during posthospital transition. J Gen Intern Med 2014;29:283-9.

\section{Implications for practice and research}

- Hospitals and providers need a deeper understanding of socioeconomic factors that are related to poor outcomes after hospital discharge to better serve patients with low socioeconomic status (SES).

- Efforts to involve low-SES patients in their hospital care, such as through collaborative goal setting, should be considered.

- Research on the relation between patient self-efficacy, adherence to recommended posthospital behaviours and long-term outcomes is needed.

\section{Context}

Studies have found that patients attribute poor posthospital transitions to lack of preparedness, sense of exclusion from decisions regarding their care plan, abandonment by the healthcare system after discharge and lack of adherence to discharge recommendations. These concerns are further complicated by low SES. This study sought to determine the experience of low-SES patients during posthospital transition.

\section{Methods}

Semistructured interviews were conducted with 65 low-SES patients to ask about their hospital experience, the perceived importance of recommended posthospital health behaviours and reported socioeconomic factors that may have influenced their adherence to recommended health behaviours. A modified grounded theory approach was used, in which all coding was performed by two research assistants and discussed with other members of the study team. Feedback on results was sought from patients who participated in the study and from the communities in which they lived.

\section{Findings}

Six themes were identified from the narratives of low-SES patients about their hospitalisation, discharge and posthospital transition. These were powerlessness during hospitalisation, misalignment of patient and care team goals, lack of saliency of health behaviours, socioeconomic constraints on patients' ability to perform recommended behaviours, abandonment by social supports and the healthcare system, and loss of self-efficacy resulting from failure. Together, these findings suggest many low-SES patients report feeling disconnected from their providers and do not feel empowered over their care.

\section{Commentary}

Adults with low SES are known to be at a greater risk for a range of physical and mental health problems than those with higher SES. ${ }^{12}$ This study provides insight on some of the problems that low-SES patients report experiencing when transitioning out of hospital care and when following recommendations for posthospital care. Almost by definition, low-SES patients likely do not have as many material resources, which may influence their posthospital outcomes. But what is particularly worth underscoring in the study findings are the subjective feelings of powerlessness, abandonment and disempowerment reported by low-SES patients.

A qualitative design was appropriate for this topic as it would have been hard to capture some of the issues mentioned by patients. However, additional research is needed to corroborate the problems reported by patients and to systematically examine patient posthospital behaviours. The complex relation between SES and race, which has been found to be related to health service utilisation in the USA was also not examined. ${ }^{3}{ }^{4}$

It is possible that many higher SES patients have similar concerns regarding their hospital care. Nonetheless, the main implication remains that involving patients in their own hospital and posthospital care may empower them and lead to better outcomes, particularly for those with low SES. Hospitals should carefully consider how to do this, which may include aspects of patient education, collaborative goal setting and rapport building. Perhaps lessons can be taken from the mental health field, where a large movement towards client-centred, recovery-oriented care has occurred in the past two decades. ${ }^{5} 6$

There may also be systemic issues to consider. It may not only be the SES of patients that limits their ability to be healthy after hospital discharge-hospitals may lack the resources and personnel to adequately engage these patients. Hospitals that serve predominantly low-income patients with public funds are often bogged down by financial constraints and other pressing priorities. Thus, the care of low-SES patients may need to be addressed at multiple levels.

Competing interests None.

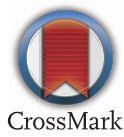

\section{References}

1. Krieger N, Chen JT, Waterman PD, et al. Painting a truer picture of US socioeconomic and racial/ethnic health inequalities: the Public Health Disparities Geocoding Project. Am J Public Health 2005;95:312-23.

2. Lantz PM, House JS, Lepkowski JM, et al. Socioeconomic factors, health behaviors, and mortality: results from a nationally representative prospective study of US adults. JAMA 1998;279:1703-8.

3. Tsai J, Desai MU, Cheng AW, et al. The effects of race and other socioeconomic factors on health service use among a nationally representative sample of U.S. veterans. Psychiatr Q 2014;85:35-47.

4. Williams DR, Mohammed SA, Leavell J, et al. Race, socioeconomic status, and health: complexities, ongoing challenges, and research opportunities. Ann N Y Acad Sci 2010;1186:69-101.

5. Tsai J, Salyers MP. Recovery orientation in hospital and community settings. J Behav Health Serv Res 2010;37:385-99.

6. Anthony WA. Recovery from mental illness: the guiding vision of the mental health service system in the 1990s. Psychiatr Rehabil J 1993;16:11-23. 